\title{
Optimization of Verapamil Drug Analysis by Excitation-Emission Fluorescence in Combination with Second-order Multivariate Calibration
}

\author{
João M. M. Leitão • Joaquim C. G. Esteves da Silva • \\ Ana Jiménez Girón • Arsenio Muñoz de la Peña
}

Received: 15 January 2008 / Accepted: 18 February 2008/Published online: 11 March 2008

(C) Springer Science + Business Media, LLC 2008

\begin{abstract}
Excitation emission fluorescence matrices (EEMs) of Verapamil drug were obtained by direct and by derivatization fluorescence spectroscopy. The fluorescence excitation and emission wavelengths were displaced to longer wavelengths and the fluorescence intensity was enhanced upon derivation with respect to the native fluorescence of the drug. The complete EEM of the native fluorescence of the drug and of the derivatization product were rapidly acquired by using a charged-coupled device detector (CCD), which is advantageous in terms of speed in the analysis, with respect to the use of a conventional photomultiplier detector. The EEMs were analyzed by several second-order multivariate calibration methods exploiting the second order advantage. The three-dimensional decomposition methods used, based in different assumptions about the trilinearity of the three
\end{abstract}

J. M. M. Leitão

Centro de Estudos Farmacêuticos,

Laboratório de Métodos Instrumentais de Análise,

Faculdade de Farmácia da Universidade de Coimbra,

3000-432, Coimbra, Portugal

J. C. G. Esteves da Silva $(\square)$

Centro de Investigação em Química, Departamento de Química,

Faculdade de Ciências da Universidade do Porto,

R. Campo Alegre 687,

4169-007, Porto, Portugal

e-mail: jcsilva@fc.up.pt

A. J. Girón · A. Muñoz de la Peña

Departamento de Química Analítica,

Universidad de Extremadura,

06071 Badajoz, Spain way data structure under analysis, were parallel factor analysis (PARAFAC), bilinear least squares (BLLS), parallel factor analysis 2 (PARAFAC2) and multivariate curve resolution-alternating least squares (MCR-ALS). The determination was performed by using the standard addition approach. The figures of merit of the PARAFAC and BLLS methods were calculated, obtaining a lower limit of detection with the derivatization procedure, when compared with the direct measurement of the fluorescence of the drug. In Verapamil drug the best estimations were found with the BLLS and the MCR-ALS models. In the quantification of Verapamil in a pharmaceutical formulation the best estimation, when compared with the result obtained by the US Pharmacopeia high performance liquid chromatography approach, was obtained by direct fluorescence spectroscopy with MCR-ALS and by derivatization fluorescence spectroscopy with the PARAFAC2 model.

Keywords Verapamil drug · Pharmaceutical formulation . Direct and derivatization fluorescence EEM - PARAFAC . BLLS · PARAFAC2 $\cdot$ MCR-ALS

\section{Introduction}

Spectrofluorimetry is a well known analytical technique used as a quantitative tool in several areas, such as chemistry, medicine, environmental and food science, owing to its intrinsic sensitivity, easiness of use and availability of instruments [1,2]. Nevertheless, traditional fluorescence spectra are broad and featureless and analyte spectra are often irresolvable from interferent spectra or background fluorescence. In consequence, the analysis of multi-component 
or complex samples can be hindered when the measurements are carried out in only one excitation or emission wavelength, and, in these cases, high order data are particularly suitable.

Nowadays, the availability of high-order instrumental data, coupled to newly chemometric algorithms for data processing, is increasing the regular use of multi-way analysis for complex analytical problems [3, 4]. Second order, i.e., matrix data for a given sample, can be produced in a variety of ways, among which one of the simpler is an excitation-emission matrix (EEM). It is well known that molecular fluorescence generates multidimensional excitation-emission spectral data per sample and that the recording of this type of data conveys the advantage of been carried out in a single instrument. EEMs present simultaneously all the excitation and emission spectra of one sample, and can be defined as a three dimensional plot with the excitation and emission wavelengths and corresponding intensities as $x y z$ axes. Alternatively, EEMs can be represented as a numerical data matrix of the fluorescence intensities as function of the excitation and emission wavelengths. Because EEMs contain a lot of information about the sample under analysis they are particularly useful for the analysis of complex mixtures [2]. Examples on the use of second-order spectrofluorimetric data to analyze complex samples can be found in recent reports $[4,5]$.

A drawback for the use of the full potential of the information content in the EEMs is the time needed to obtain one data matrix, if a conventional photomultiplier detection system is used. Nowadays, a solution to this problem is the use of a spectrofluorimeter equipped with a charged-coupled device detector (CCD), that allows fast acquisition of full EEMs [1, 2]. In consequence, the outlined advances in molecular fluorescence instrumentation and chemometrics three-dimensional decomposition methods make feasible the use of multi-parametric determinations to achieve selective measurements [3, 4]. The rapid acquisition, coupled to the use of three-dimensional decomposition methods, gives these analytical methodologies a great potential for their application on rotinary quality analytical control [5].

However, fluorescence based analytical measurements may have some limitations due to: chemical interferences; Rayleigh or Raman scattering; overlapped fluorescence bands; fluorescence background or instrumental noise [1, 2, 6]. Nevertheless, fluorescence is inherently linear in all dimensions [7]. An excitation-emission matrix consists of a trilinear part plus some random (noise) and minor deviations from the trilinearity due to scattering and other chemical and/or instrumental interferences [8]. The latter are also called three-way data, and are characterized by following the trilinear or PARAFAC model. Interestingly, the decomposition of a three-dimensional cube of data is often unique. This is due to the fact that three-way data are not merely a collection of two-way data sets, but there is actually an internal relationship between each of the matrices. As a consequence, the decomposition of a threeway data array built with response matrices measured for a number of samples, allows emission and excitation spectral profiles, as well as relative concentrations of individual sample components to be extracted directly. If the model is correctly specified with the right number of components, and the trilinear structure is approximately valid, then the PARAFAC solution will provide estimates of the underlying parameters. This property has been named the secondorder advantage [9]. Hence, the combination of EEMs with multi-way calibration methods, achieving the second-order advantage, makes possible the correct modeling of the data in the presence of possible chemical and/ or instrumental interferences, and can minimize or eliminate traditionally time-consuming sample pretreatments.

Verapamil is a calcium channel blocker widely used as an antihypertensive, antianginal and antiarhythmic drug [10]. When excited with ultraviolet light exhibits fluorescence in different solvents. In a previous work, the adequate quantification of Verapamil drug, by measuring the direct native fluorescence of the drug, using a spectrofluorimeter with a photomultiplier detector, was demonstrated to be feasible by PARAFAC calibration [11].

It has been demonstrated that, by catalysis of the tertiary amino group present in his molecule, by means of the reaction of malonic acid with acetic anhydride, a fluorescence derivate is obtained at higher wavelengths than the intrinsic fluorescence of Verapamil. In this work, several basic objectives were pretended, a substantial increment of the speed of the analysis, better chemical conditions to perform the Verapamil determination and the use of several second-order multivariate calibration algorithms, allowing an enhancement of the prediction results. In this context, EEMs obtained by direct and by derivatization fluorescence, using a spectrofluorimeter with a CCD detector, will be analyzed with three-dimensional decomposition methods, namely: parallel factor analysis (PARAFAC); bilinear least squares (BLLS); parallel factor analysis 2 (PARAFAC2) and multivariate curve resolution-alternating least squares (MCR-ALS).

The PARAFAC $[12,13]$ and BLLS $[14,15]$ models deal with trilinear three-way data, PARAFAC2 model $[16,17]$ handle with small deviations from trilinearity in one of the dimensions and with different rows (or columns) dimensions of the slices in the three-way data and MCR-ALS model [18] deals with non trilinear three-way data. The recently introduced BLLS algorithm, is an alternative to PARAFAC, also exploiting the second-order advantage. These chemometric methods are being used in analytical methodologies coupled to EEM measurements, namely: 
environmental studies [19-28]; clinical analysis [11, 2932]; and food research [33, 34].

The analytical performance of PARAFAC, BLLS, PARAFAC2 and MCR-ALS, to quantify Verapamil drug, from EEMs obtained by direct and by derivatization fluorescence spectroscopy, will be compared using standard solutions and one pharmaceutical formulation. Also, the results obtained will be compared with those obtained by a reference US Pharmacopeia high performance liquid chromatography with ultraviolet detection method (USP HPLC-UV).

\section{Experimental}

Reagents and solutions

Verapamil reference standard and Malonic acid (both $\geq 99 \%$ purity) were obtained from Sigma-Aldrich Química S.A. (Spain). Anhydride acetic, methanol and ethanol were purchased from Merck, Darmstadt (Germany). Deionised water with resistivity higher than $4 \mathrm{M} \Omega / \mathrm{cm}$ was used. The pharmaceutical formulation under analysis was Isoptin ${ }^{\circledR}$ injectable solution $(5 \mathrm{mg} / 2 \mathrm{ml})$ from Knoll.

The Verapamil standard stock solutions were prepared in methanol (direct fluorescence spectroscopy) or in ethanol (derivatization fluorescence spectroscopy) by rigorous weighting. For the preparation of the pharmaceutical formulation stock solution the volume of a vial was rigorously diluted to obtain the required concentration.

\section{Procedures}

For Verapamil direct fluorescence spectroscopy the standard concentrations were obtained using successive three $10 \mu \mathrm{l}$ additions of a $750 \mathrm{ppm}$ standard solution to $3 \mathrm{ml}$ of a $5 \mathrm{ppm}$ standard or sample solution contained in a $1 \mathrm{~cm}$ quartz cell - to obtain concentrations increments of $2.5,5$ and $7.5 \mathrm{ppm}$.

For Verapamil derivatization fluorescence spectroscopy the dilution of the 1,000 ppm Verapamil standard or sample solution to an intermediate $10 \mathrm{ppm}$ solution was done in a way as to obtain the fluorescent derivate condensation product. For a final volume of $10 \mathrm{ml}$ the dilution was done after solvent evaporation, addition of $2 \mathrm{ml}$ malonic acid reagent $0.5 \%$, promotion of the reaction at $100{ }^{\circ} \mathrm{C}$ during $5 \mathrm{~min}$ followed by dilution to $10 \mathrm{ml}$. The concentration of the diluted standard or sample solutions of the fluorescent derivate condensation product in $5.00 \mathrm{ml}$ glass volumetric flasks was $0.3 \mathrm{ppm}$. As for the Verapamil direct fluorescence spectroscopy three additions of the $10 \mathrm{ppm}$ standard solution were done in order to obtain standard concentrations of $0.1,0.3$, and $0.5 \mathrm{ppm}$.
For the chromatographic quantification the mobile phase, standard and sample solutions were prepared following the USP recommendations [10]. Standards were prepared in the mobile phase by rigorous weighting to a final concentration of 2,000 ppm. Diluted standards and sample solutions were obtained by dilution with methanol to the required concentrations. Standard solutions in the range from 50 to $500 \mathrm{ppm}$ and an expected concentration of $250 \mathrm{ppm}$ for the pharmaceutical formulation sample solution were used.

Instrumentation

A Spex 3D Spectrofluorimeter with a $75 \mathrm{~W}$ xenon lamp with a CCD detector was used. EEM were acquired, in an excitation wavelength range from 250 to $550 \mathrm{~nm}$, and in an emission wavelength range from 250 to $710 \mathrm{~nm}$, with a resolution of $5 \mathrm{~nm}$ and one accumulation. For the direct fluorescence measurements, an integration time of $18 \mathrm{~s}$ (slits with $0.05 \mathrm{~mm}$ ) was used and, for the derivatization fluorescent measurements, an integration time of $0.4 \mathrm{~s}$ (slits with $0.1 \mathrm{~mm}$ ) were used.

The HPLC determinations were made with a system constituted by a HP1100 isocratic pump, a manual injection valve Rheodyne, model 7752i, a Rheodyne $20 \mu \mathrm{L}$ loop, a pre-column Agilent $(20 \times 4 \mathrm{~mm})$ and a Supelco column $(100 \times 4.6 \mathrm{~mm})$ Hypersil $^{\circledR}$ ODS of $3 \mu \mathrm{m}$ particle diameter and a diode array detector (DAD) Ati Unicam Crystal 250 model. The DAD spectra were acquired in a wavelength range from 200 to $452 \mathrm{~nm}$ with a resolution of $2 \mathrm{~nm}$. The wavelength used for the quantification was $280 \mathrm{~nm}$.

\section{Chemometric analysis}

The matrices of experimental data points [excitation $(\mathrm{nm}) \times$ emission (nm)] obtained at different standard concentrations were analyzed using the PARAFAC, BLLS, PARAFAC2 and MCR-ALS and implemented in MATLAB code. The PARAFAC, PARAFAC2 and MCR-ALS estimations are found by an iterative alternating least squares procedure. As convergence criteria a value of $1 \times 10^{-6}, 1 \times 10^{-7}$ and $1 \times 10^{-3}$ and a maximum number of iterations 2,500, 2,000 and 50 were used for PARAFAC, PARAFAC2 and MCR-ALS, respectively. The initial estimates used for the PARAFAC, PARAFAC2 and the MCR-ALS were the estimates of a model without constraints. The BLLS estimations are found by a direct least-squares procedure. No initialization or constraint procedures are required.

For the analysis with PARAFAC and BLLS the data sets are structured as [excitation $(\mathrm{nm}) \times$ emission $(\mathrm{nm}) \times$ concentration], for the analysis with PARAFAC2 the data sets are structured as [emission $(\mathrm{nm}) \times$ excitation $(\mathrm{nm}) \times$ concentration] and for the analysis with MCR-ALS as 
[(concentration $\times$ emission $(\mathrm{nm})) \times$ excitation $(\mathrm{nm})]$. Except for PARAFAC2 on the second dimension, non-negativity constraints are applied in all the dimensions of the Verapamil drug matrices analyzed.

Raw EEMs were reduced for chemometric analysis (Fig. 1). EEMs obtained by direct spectroscopy were reduced for an excitation wavelength range from 251.70 to $301.83 \mathrm{~nm}$ (10 wavelengths) and an emission wavelength range from 252.27 to $548.65 \mathrm{~nm}$ (58 wavelengths). EEMs obtained by derivatization spectroscopy were reduced for an excitation wavelength range from 251.70 to $452.23 \mathrm{~nm}$ (37 wavelengths) and an emission wavelength range from 397.86 to $600.64 \mathrm{~nm}$ (40 wavelengths).

All routines employed to carry out the calculations described in this paper were written in MATLAB [35]. Those for applying PARAFAC and PARAFAC2 models are available from http://www.models.kvl.dk/source/

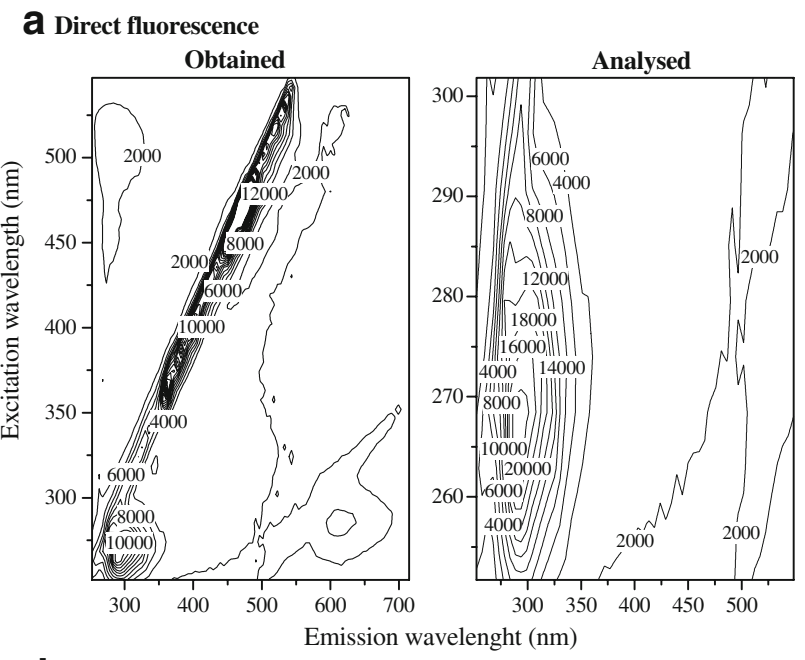

b Derivatization fluorescence - malonic acid / anhydride acetic

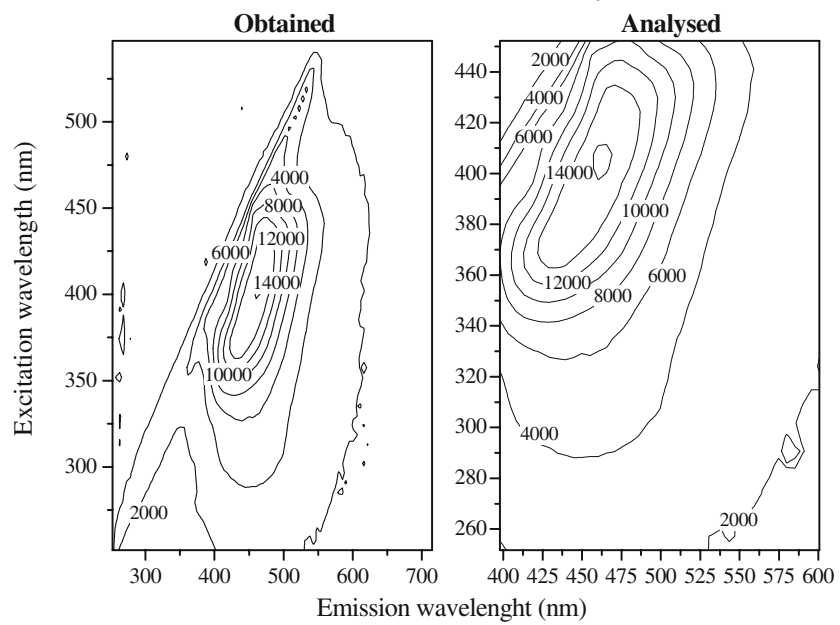

Fig. 1 Raw and analysed Verapamil drug EEM. Obtained in a by direct fluorescence $(4.98 \mathrm{ppm})$ and in $\mathbf{b}$ by derivatization fluorescence spectroscopy $(0.30 \mathrm{ppm})$ nwaytoolbox/ and the MCR-ALS program is available from http://www.ub.es/gesq/mcr/. For BLLS and for the calculation of the figures of merit with both PARAFAC and BLLS models, the MVC-2 routine, available from the authors on request, was used, including a useful graphical interface for data input and parameter settings, of the type already described for first order calibration [36].

Multi-way model fit analysis

The results obtained from the different PARAFAC, BLLS, PARAFAC2 and MCR-ALS models were compared using the model fit [Fit (\%)]. The model fit (\%) for PARAFAC and PARAFAC2 models is defined by Eq. 1 .

$\operatorname{Fit}(\%)=100 \times\left(1-\sqrt{\frac{\sum_{i=1}^{I} \sum_{j=1}^{J} \sum_{k=1}^{K}\left(x_{i j k}-\widehat{x}_{i j k}\right)^{2}}{\sum_{i=1}^{I} \sum_{j=1}^{J} \sum_{k=1}^{K}\left(x_{i j k}\right)^{2}}}\right)$

In this equation $\widehat{x}_{i j k}$ is the $i j k$ element of the estimated three-dimensional matrix and $x_{i j k}$ is the $i j k$ element of the experimental three-dimensional matrix. The model fit (\%) for MCR-ALS models is defined by Eq. 2.

$\operatorname{Fit}(\%)=100 \times\left(1-\sqrt{\frac{\sum_{l=1}^{L} \sum_{j=1}^{J}\left(x_{l j}-\hat{x}_{l j}\right)^{2}}{\sum_{l=1}^{L} \sum_{j=1}^{J}\left(x_{l j}\right)^{2}}}\right)$

In this equation $\widehat{x}_{l j}$ is the $l j$ element of the estimated bidimensional column-wise augmented data matrix and $x_{l j}$ is the $l j$ element of the experimental bidimensional columnwise augmented data matrix with $l=k \times i$.

Also, the results obtained with PARAFAC models were assessed using the CORCONDIA or core consistency test [Corcondia(\%)] defined by Eq. 3 [8, 12].

$\operatorname{Corcondia}(\%)=100 \times\left(1-\frac{\sum_{d=1}^{N} \sum_{e=1}^{N} \sum_{f=1}^{N}\left(g_{d e f}-t_{d e f}\right)^{2}}{\sum_{d=1}^{N} \sum_{e=1}^{N} \sum_{f=1}^{N}\left(t_{d e f}\right)^{2}}\right)$

In this equation $g_{\text {efg }}$ and $t_{\text {efg }}$ denote the elements of the calculated core and of the intrinsic super-diagonal core, respectively, and $N$ the number of components of the model. If they are equal, the core consistency is perfect and has a value of unity $(100 \%)$. The appropriate number of components is accessed with the model with the highest number of components and a valid value of core consistency diagnostic test. Usually, the number of components is 
taken as the largest number for which the latter parameter is larger than around 50 .

The results obtained with MCR-ALS models were assessed using the lack of fit [LOF(\%)] of the MCR-ALS model defined by Eq. 4 [18].

$\operatorname{LOF}(\%)=100 \times\left(\sqrt{\frac{\sum_{l=1}^{L} \sum_{j=1}^{J}\left(x_{l j}-\widehat{x}_{l j}\right)^{2}}{\sum_{l=1}^{L} \sum_{j=1}^{J}\left(x_{l j}\right)^{2}}}\right)$

In this equation $\widehat{x}_{l j}$ is the $l j$ element of the estimated bidimensional column-wise augmented data matrix by the MCR-ALS model and $x_{l j}$ is the $l j$ element of the experimental column-wise augmented data matrix in the $\mathrm{LOF}_{\text {MCR }}$ vs Exp calculus and of the estimated with principal component analysis in the $\mathrm{LOF}_{\mathrm{MCR}}$ vs PCA calculus.

Figures of merit

For both linear models, PARAFAC and BLLS, the figures of merit were also evaluated. Two important sensitivity equations have been discussed in the literature, both based on extensions of Lorber's net analyte signal (NAS) theory [37] to the second-order domain. More useful equations are obtained by considering that the various high-order chemometric methods provide estimated component profiles in the different dimensions. In the present context, a useful sensitivity parameter is the so-called MKL (after Messick et al. [38]) sensitivity for constituent $n$, whose mathematical expression as a function of the component profiles, is [39]:

$\mathrm{SENn}, \mathrm{MKL}=k_{n}\left\{\left[\left(\boldsymbol{B}^{T} \boldsymbol{B}\right) *\left(\boldsymbol{C}^{T} \boldsymbol{C}\right)\right]^{-1}\right\}_{n n}^{-1 / 2}$

where $\mathbf{B}$ and $\mathbf{C}$ are matrices containing, as columns, the profiles for all components in the first and second dimension respectively (all normalized to unit length), ' $n n$ ' designates the $(n, n)$ element of a matrix, $k_{n}$ is the total signal for component $n$ at unit concentration, and the symbol ' $*$ ' is the element-wise Hadamard product matrix. This MKL approach to NAS has already been rigorously proved to be connected to cases where the second-order advantage is not exploited, for example, when employing PARAFAC [8], or BLLS with the so-called least-squares predictor [40].

In Eq. 5, the profiles are provided with three-way decomposition using the appropriate algorithm, while the value of $k_{n}$ is also computed by the algorithm, using information on the nominal calibration concentrations for component $n$.

The selectivity is obtained by dividing the sensitivity by the total signal for the pure component, and therefore a suitable expression is [14]:

SELn, MKL $=\left\{\left[\left(\mathbf{B}^{\mathrm{T}} \mathbf{B}\right) *\left(\mathbf{C}^{\mathrm{T}} \mathbf{C}\right)\right]^{-1}\right\}_{n n}^{-1 / 2}$

More useful than the sensitivity appears to be the analytical sensitivity $\gamma_{n}$, defined, in analogy with univariate calibration, as the quotient between the sensitivity and the instrumental noise level. Its inverse establishes the minimum concentration difference which can be appreciated across the linear range, and is independent on instrument or scale [41].

The limit of detection $\left(L O D_{n}\right)$ should also be considered. An approximation to the $L O D_{n}$ can be gathered from the expression:

$\mathrm{LOD}_{n}=3 s_{X} / \mathrm{SEN}_{n}$

where $s_{X}$ is an estimate of the instrumental noise level.

\section{Results and discussion}

Figure 1 shows typical raw and reduced EEMs obtained with direct and derivatization fluorescence spectroscopy of Verapamil. The analysis of this figure shows that both EEMs contain first order scattering which interferes with the main fluorescence band. In the EEM obtained by direct fluorescence it is also possible to verify the existence of the minor second order scattering bands. In the EEM obtained by derivatization fluorescence spectroscopy there is a shift towards higher wavelengths of the excitation and emission
Table 1 Explained variance of the first five components obtained by SVD

[exc. $\times($ conc.$\times$ emi. $)]$ Matrix row augmented in which the rows are the excitation and the columns are the emission spectra obtain in the four concentrations levels

\begin{tabular}{|c|c|c|c|c|}
\hline \multirow{2}{*}{$\begin{array}{l}\text { Principal } \\
\text { component }\end{array}$} & \multicolumn{2}{|c|}{ Direct fluorescence } & \multicolumn{2}{|c|}{ Derivatization fluorescence } \\
\hline & $\begin{array}{l}\text { Verapamil, } \\
5 \text { ppm }\end{array}$ & $\begin{array}{l}\text { Four standards } \\
{[\text { exc. } \times(\text { conc } \times \text { emi. })]}\end{array}$ & $\begin{array}{l}\text { Verapamil, } \\
0.3 \mathrm{ppm}\end{array}$ & $\begin{array}{l}\text { Four standards } \\
{[\text { exc. } \times(\text { conc } \times \text { emi. })]}\end{array}$ \\
\hline 1 & 97.87 & 98.55 & 93.63 & 90.54 \\
\hline 2 & 1.30 & 0.87 & 5.34 & 8.09 \\
\hline 3 & 0.50 & 0.43 & 0.88 & 1.20 \\
\hline 4 & 0.18 & 0.07 & 0.07 & 0.11 \\
\hline 5 & 0.08 & 0.04 & 0.04 & 0.04 \\
\hline
\end{tabular}




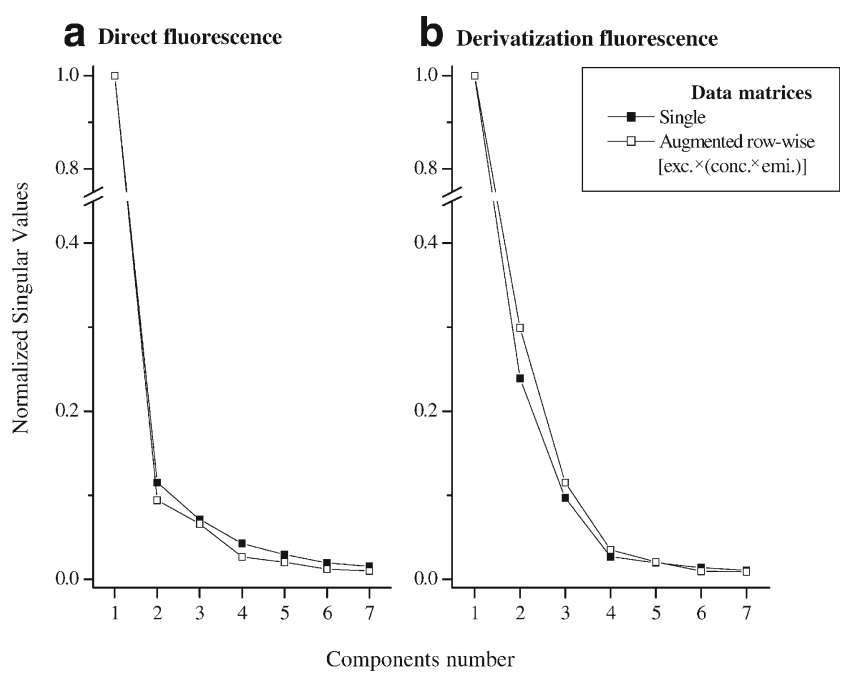

Fig. 2 Singular value decomposition of single and augmented rowwise data matrices of Verapamil drug obtain in a by direct fluorescence $(4.98 \mathrm{ppm})$ and in $\mathbf{b}$ by derivatization fluorescence spectroscopy $(0.30 \mathrm{ppm})$

bands. Indeed, derivatization with malonic acid/anhydride acetic provokes a displacement of the excitation maximum from 375 to $425 \mathrm{~nm}$ and a displacement of the emission maximum from 425 to $475 \mathrm{~nm}$.
The strategy used in this work to reduce the effect of the scatter bands was to eliminate the section of the EEMs that contain them. However, it was not possible to separate completely the scatter bands from the main fluorescence bands - this is particular critical in the direct fluorescence EEMs. This information must be accounted for when the number of linear independent factors present in the EEMs are estimated.

Interestingly, it is clear from Fig. 1 that the derivatization approach is advantageous over the direct approach, as the excitation and emission maxima of the derivatized product are more separated from the scatter bands than in the direct procedure.

Singular value decomposition (SVD)

Table 1 and Fig. 2 show the results of the SVD of the single and augmented row-wise [excitation $\times($ concentration $\times$ emission)] data matrices. In Fig. 2, for an easier comparison, normalized singular values for the single and augmented row-wise matrix are used.

From the analysis of the explained variance of the singular values of the augmented EEM (Table 1) it is shown

Table 2 Comparison of the results obtained by the PARAFAC, BLLS, PARAFAC2 and MCR-ALS models in the analysis of a Verapamil drug EEM (4.98 ppm) obtained by direct fluorescence spectroscopy

\begin{tabular}{|c|c|c|c|c|c|}
\hline \multirow[t]{2}{*}{ Model evaluation } & \multicolumn{5}{|c|}{ Number of components } \\
\hline & Two & Three & Four & Five & Six \\
\hline \multicolumn{6}{|c|}{ Fluorescence at maximum fluorescence intensity (excitation $263 \mathrm{~nm}$ and emission $289 \mathrm{~nm}$ ) } \\
\hline \multicolumn{6}{|c|}{ Estimated concentration $(\mathrm{ppm})=5.71(0.06)$, Recovery $=114.7 \%$} \\
\hline \multicolumn{6}{|c|}{ PARAFAC } \\
\hline Estimated concentration (ppm) & $4.83(0.21)$ & $4.94(0.20)$ & $5.40(0.10)$ & $5.46(0.13)$ & $5.44(0.11)$ \\
\hline Recovery $(\%)$ & 97.0 & 99.2 & 108.4 & 109.6 & 109.2 \\
\hline Fit $(\%)$ & 91.7 & 94.8 & 95.8 & 93.6 & 94.5 \\
\hline Number of Iterations & 38 & 120 & 686 & 2500 & 2500 \\
\hline Corcondia (\%) & 98.1 & -0.3 & 4.6 & 11.4 & 40.6 \\
\hline \multicolumn{6}{|l|}{ BLLS } \\
\hline Estimated concentration (ppm) & $4.97(0.11)$ & $4.97(0.068)$ & $5.21(0.064)$ & $8.31(0.95)$ & $5.00(0.072)$ \\
\hline Recovery $(\%)$ & 99.8 & 99.8 & 104.6 & 166.9 & 100.4 \\
\hline Number of Iterations & 89 & 96 & 384 & 6614 & 5374 \\
\hline \multicolumn{6}{|l|}{ PARAFAC2 } \\
\hline Estimated concentration (ppm) & $6.02(0.05)$ & $4.76(0.25)$ & $8.74(1.45)$ & $9.11(1.46)$ & $5.33(0.21)$ \\
\hline Recovery $(\%)$ & 120.9 & 95.5 & 175.5 & 182.9 & 107.0 \\
\hline Fit $(\%)$ & 91.7 & 94.9 & 96.1 & 96.9 & 97.7 \\
\hline Number of Iterations & 2000 & 755 & 2000 & 2000 & 2000 \\
\hline \multicolumn{6}{|l|}{ MCR-ALS } \\
\hline Estimated concentration (ppm) & $4.99(0.22)$ & $4.82(0.15)$ & $4.82(0.14)$ & $5.18(0.21)$ & $4.84(0.21)$ \\
\hline Recovery $(\%)$ & 100.2 & 96.8 & 96.8 & 104.0 & 97.2 \\
\hline Fit $(\%)$ & 92.4 & 96.2 & 97.2 & 98.0 & 98.5 \\
\hline Number of Iterations & 50 & 50 & 50 & 50 & 50 \\
\hline Lof $_{\text {MCRvs.PCA }}(\%)$ & 0.02 & 0.03 & 0.18 & 0.55 & 0.55 \\
\hline Lof $_{\text {MCRvsExp. }}(\%)$ & 7.59 & 3.81 & 2.77 & 1.96 & 1.55 \\
\hline
\end{tabular}

For the estimated concentration the values in brackets are the standard deviation of the extrapolated concentration 

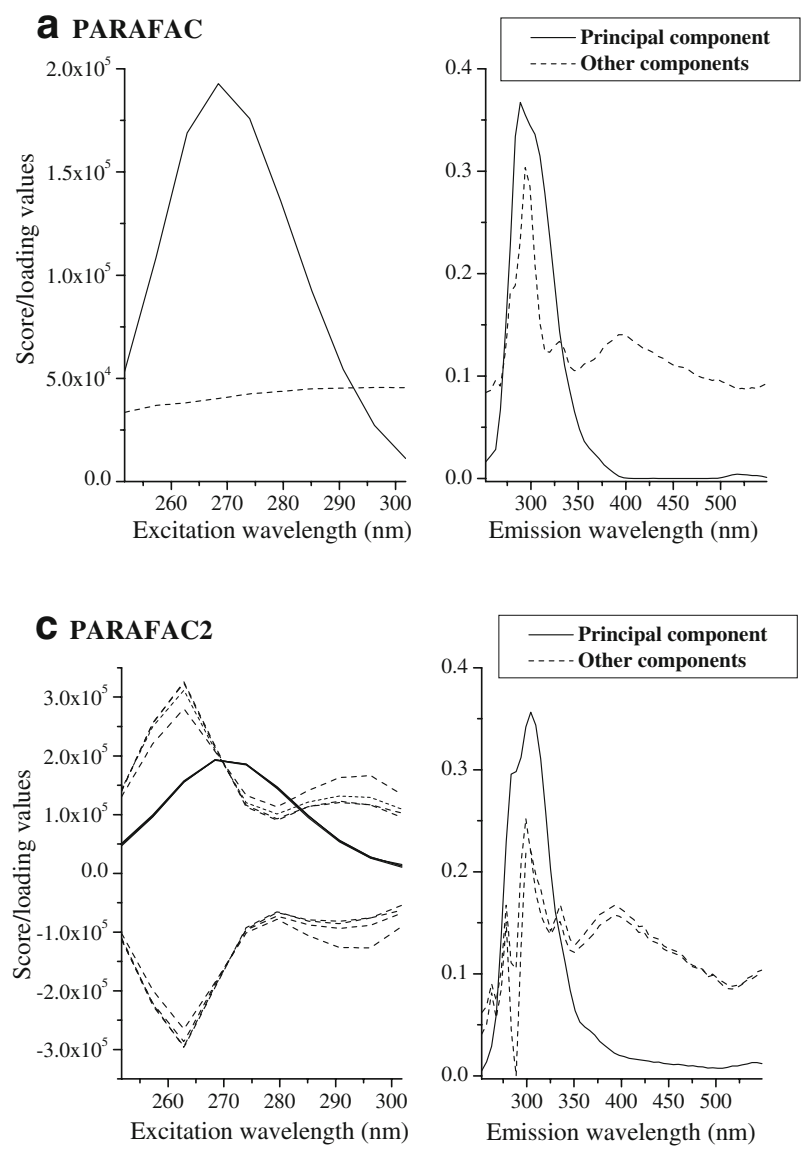

Fig. 3 Excitation and emission spectra estimated from the analysis of the Verapamil drug EEM obtained by direct fluorescence: a two components non-negativity constraint PARAFAC model, b one component and one interferent BLLS model, $\mathbf{c}$ three components

that two components explain almost $99 \%$ of variance. From the analysis of the normalized singular values of the single and augmented row-wise matrices (Fig. 2), a minimum of two and a maximum of five components may be expected for the single and augmented data matrices of the direct and derivatization EEM. It is known that, when there is scattering superimposed with the fluorescence signal, several minor components appear to explain the spectral variance. Taking into consideration this information, these numbers of components will be considered in the subsequent analysis by PARAFAC, BLLS, PARAFAC2 and MCR-ALS.

Three-dimensional decomposition analysis

\section{Direct fluorescence spectroscopy}

Table 2 presents the results obtained using the fluorescence intensities at the maximum emission (excitation at $263 \mathrm{~nm}$ and emission at $289 \mathrm{~nm}$ ) and with PARAFAC, BLLS,

\section{b BLLS}
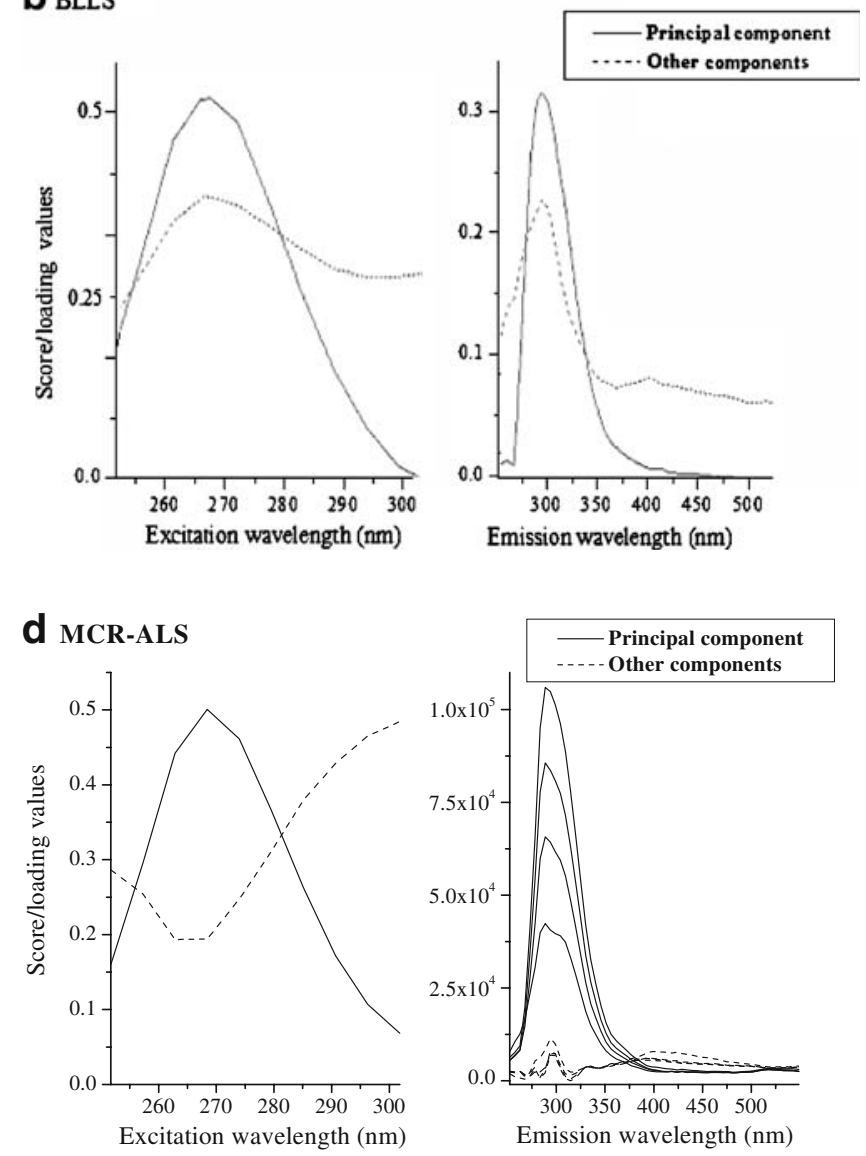

non-negativity constraint in the first and third dimensions PARAFAC2 model and $\mathbf{d}$ two components non-negativity constraint MCR-ALS model

PARAFAC2 and MCR-ALS modelling of the direct EEM. The analysis of Table 2 shows that readings at maximum fluorescence intensity overestimates the concentration of Verapamil (recovery $=115 \%$ ) and only multivariate models allow good concentrations estimations.

The fit achieved with MCR-ALS are always higher than the fit obtained with PARAFAC and PARAFAC2. Similar

Table 3 Comparison of the figures of merit calculated for the PARAFAC and BLLS models at the number of components indicated obtained by direct fluorescence spectroscopy

\begin{tabular}{|c|c|c|}
\hline & PARAFAC & BLLS \\
\hline Number of components & 2 & 2 \\
\hline 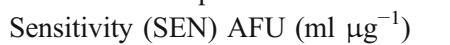 & 8,650 & 4,937 \\
\hline Analytical sensitivity $(\gamma)\left({\left.\mathrm{ml} \mu \mathrm{g}^{-1}\right)}^{-1}\right.$ & 10.0 & 8.6 \\
\hline$\gamma^{-1}\left(\mu \mathrm{g} \mathrm{ml}^{-1}\right)$ & 0.10 & 0.11 \\
\hline Selectivity (SEL) & 0.42 & 0.23 \\
\hline Limit of detection (LOD) $\left(\mu \mathrm{g} \mathrm{ml}^{-1}\right)$ & 0.30 & 0.38 \\
\hline
\end{tabular}

$A F U$ Arbitrary fluorescence units 
Table 4 Comparison of the results obtained by the PARAFAC, BLLS, PARAFAC2 and MCR-ALS models in the analysis of a Verapamil drug EEM $(0.30 \mathrm{ppm})$ obtained by derivatization fluorescence spectroscopy

\begin{tabular}{|c|c|c|c|c|c|}
\hline \multirow[t]{2}{*}{ Model evaluation } & \multicolumn{5}{|c|}{ Number of components } \\
\hline & Two & Three & Four & Five & Six \\
\hline \multicolumn{6}{|c|}{ Fluorescence at maximum fluorescence intensity (excitation $408 \mathrm{~nm}$ and emission $466 \mathrm{~nm}$ ) } \\
\hline \multicolumn{6}{|c|}{ Estimated concentration $(\mathrm{ppm})=0.33(0.06)$, recovery $=110.0 \%$} \\
\hline \multicolumn{6}{|c|}{ PARAFAC } \\
\hline Estimated concentration (ppm) & $0.37(0.04)$ & $0.28(0.04)$ & $0.27(0.05)$ & $0.24(0.05)$ & $0.31(0.09)$ \\
\hline Recovery $(\%)$ & 123.3 & 93.3 & 90.0 & 80.0 & 103.3 \\
\hline Fit $(\%)$ & 87.6 & 93.9 & 96.5 & 97.2 & 97.9 \\
\hline Number of Iterations & 188 & 246 & 364 & 360 & 1592 \\
\hline Corcondia $(\%)$ & 87.6 & 32.9 & 26.9 & 92.6 & 2.5 \\
\hline \multicolumn{6}{|l|}{ BLLS } \\
\hline Estimated concentration (ppm) & $0.29(0.08)$ & $0.30(0.02)$ & $0.32(0.02)$ & $0.33(0.03)$ & $0.42(0.26)$ \\
\hline Recovery $(\%)$ & 96.7 & 100.0 & 106.7 & 110.0 & 140.0 \\
\hline Number of Iterations & 44 & 40 & 192 & 819 & 4385 \\
\hline \multicolumn{6}{|l|}{ PARAFAC 2} \\
\hline Estimated concentration (ppm) & $0.288(0.05)$ & $0.284(0.05)$ & $0.259(0.03)$ & $0.327(0.06)$ & $0.298(0.04)$ \\
\hline Recovery $(\%)$ & 96.0 & 94.7 & 86.3 & 109.0 & 99.3 \\
\hline Fit $(\%)$ & 88.5 & 96.1 & 97.7 & 98.3 & 98.7 \\
\hline Number of Iterations & 758 & 2,000 & 2,000 & 2,000 & 2,000 \\
\hline \multicolumn{6}{|l|}{ MCR-ALS } \\
\hline Estimated concentration (ppm) & $0.35(0.03)$ & $0.39(0.04)$ & $0.30(0.03)$ & $0.30(0.03)$ & $0.29(0.03)$ \\
\hline Recovery $(\%)$ & 116.7 & 130.0 & 100.0 & 100.0 & 96.7 \\
\hline Fit $(\%)$ & 88.1 & 95.5 & 97.4 & 98.2 & 98.3 \\
\hline Number of Iterations & 21 & 50 & 50 & 50 & 21 \\
\hline Lof $_{\text {MCRvs.PCA }}(\%)$ & 1.8 & 1.6 & 0.6 & 0.7 & 1.1 \\
\hline Lof $\mathrm{MCRvsExp}_{\text {. }}(\%)$ & 11.8 & 4.5 & 2.6 & 1.7 & 1.7 \\
\hline
\end{tabular}

See footnote of Table 2

model fits were found for the PARAFAC, and PARAFAC2 from two to four components. However, the analysis of the PARAFAC CORCONDIA test shows that a two component model is the most appropriate, i.e. it corresponds to the highest value $(98.1 \%)$, before the Core consistency value is lower than $50 \%$. The analysis of the lack of fit of the MCRALS shows that a two and three component models originates relatively low values for Lof $f_{\text {MCRvs.PCA }}(0.02 \%$ and $0.03 \%$, respectively).

With a two component model, as suggested by the CORCONDIA test, PARAFAC, and MCR-ALS are adequate to model the Verapamil drug EEM obtained by direct fluorescence. With these models, the predicted concentration and described recoveries of the expected Verapamil concentration (4.98 ppm) were: 4.83 and $97.0 \%$ with PARAFAC and $4.99 \mathrm{ppm}$ and $100.2 \%$ with MCR-ALS. A slightly lower Verapamil concentration estimation of $4.82 \mathrm{ppm}$ and a recovery of $96.8 \%$ was found with a three component MCR-ALS model. For two components PARAFAC2 model the predicted concentration was not good and only a three component model allows to obtain recovery of $95.6 \%$. When BLLS was applied, taken into account one com- ponent and one interferent in the system, an estimation of $4.97 \mathrm{ppm}$ and a recovery of $99.8 \%$ were found, on the same order of magnitude than MCR-ALS. A similar result was found with one component and two interferents in the system. Nevertheless, the best estimation was found with MCR-ALS.

The Fig. 3 presents the excitation and emission spectra estimated with the models that allows the best Verapamil concentration estimation: (a) with a two components non negativity constraint PARAFAC model; (b) with a one component and one interferent system BLLS model; (c) with a three components non negativity constraint in the first and third dimensions PARAFAC2 model; (d) with a two components non negativity constraint MCR-ALS model. For all the models two components correspond to the Verapamil intrinsic fluorescent and to a background signal. For the PARAFAC2 model the other component correspond to scatter bands and background signal.

The figures of merit calculated for the PARAFAC and BLLS models are shown in Table 3. By the analysis of this table it is possible to observe that similar results were found for the figures of merit by the two models. Nevertheless a 

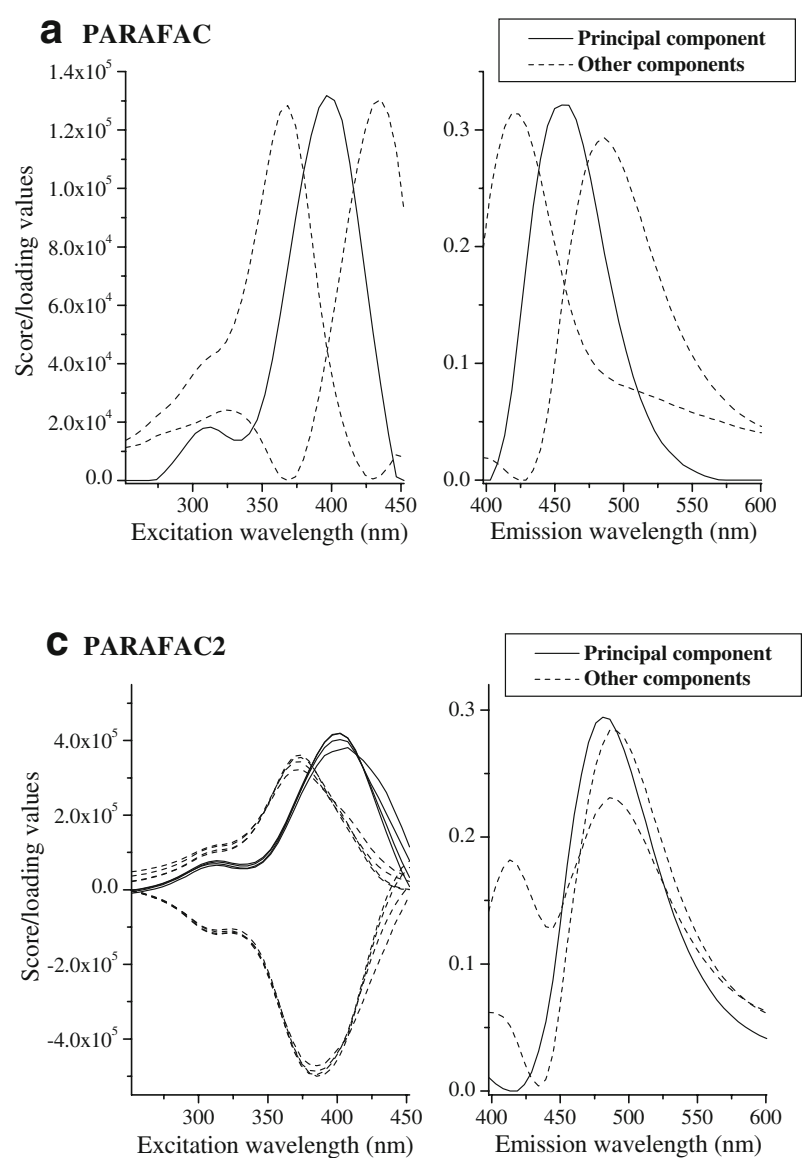

Fig. 4 Excitation and emission spectra estimated from the analysis of the Verapamil drug EEM obtained by derivatization fluorescence: a three components non-negativity constraint PARAFAC model, b one component and two interferents BLLS model, $\mathbf{c}$ three components

greater sensitivity and selectivity with a slight lower LOD were found with the PARAFAC model.

\section{Derivatization fluorescence spectroscopy}

Table 4 presents the results obtained using the fluorescence intensities at the maximum emission (excitation $408 \mathrm{~nm}$ and emission $466 \mathrm{~nm}$ ) and with PARAFAC, BLLS, PARAFAC2 and MCR-ALS modelling of the Verapamil drug EEM obtained by derivatization fluorescence spectroscopy. The analysis of Table 2 shows that readings at maximum fluorescence intensity overestimates the concentration of Verapamil (recovery $=110 \%$ ) and only multivariate models allow good concentrations estimations.

Similar models fits are achieved with the four methods, although PARAFAC originates a slightly lower fit. If the analysis of the PARAFAC CORCONDIA test is strictly applied, a two component model seems the most appropriate, i.e. it corresponds to the highest value $(87.6 \%)$ before the core consistency drops to a value lower than 50 . However,
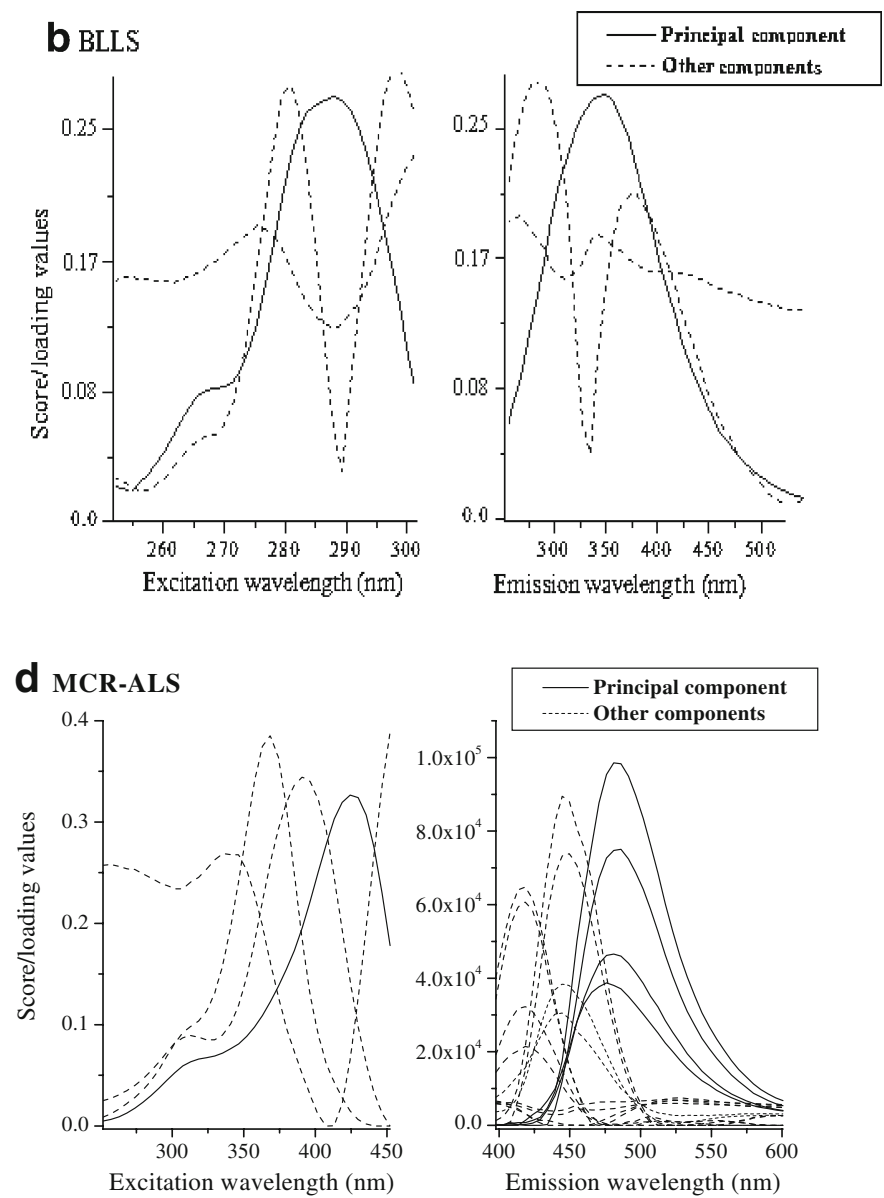

non-negativity constraint in the first and third dimensions PARAFAC2 model and $\mathbf{d}$ four components non-negativity constraint MCR-ALS model

the core consistency values of the three component model, 32.9 , and five component model of 92.6 , are indicating that more than two components may also be appropriated. The analysis of the lack of fit of the MCR-ALS models shows that with a four component model, relatively low values are obtained for Lof $_{\mathrm{MCRvs.PCA}}=0.6 \%$.

Table 5 Comparison of the merit figures calculated for the PAR AFAC and BLLS models at the number of components indicated obtained by derivatization fluorescence spectroscopy

\begin{tabular}{|c|c|c|}
\hline & PARAFAC & BLLS \\
\hline Number of components & 3 & 3 \\
\hline 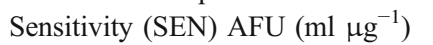 & 140,000 & 198,839 \\
\hline Analytical sensitivity $(\gamma)\left(\mathrm{ml} \mathrm{g}^{-1}\right)$ & 183 & 652 \\
\hline$\gamma^{-1}\left(\mu \mathrm{g} \mathrm{ml}^{-1}\right)$ & 0.005 & 0.002 \\
\hline Selectivity (SEL) & 0.37 & 0.34 \\
\hline Limit of detection (LOD) $\mu \mathrm{g} \mathrm{ml}^{-1}$ & 0.02 & 0.02 \\
\hline
\end{tabular}

$A F U$ Arbitrary fluorescence units 
Table 6 Comparison of the results obtained by the PARAFAC, BLLS,

PARAFAC2 and MCR-ALS models in the analysis of a Verapamil pharmaceutical formulation EEM, obtained by direct fluorescence $(4.98 \mathrm{ppm})$ and by derivatization fluorescence $(0.30 \mathrm{ppm})$ spectroscopy

See footnote of Table 2

\begin{tabular}{lclcc}
\hline Model evaluation & PARAFAC & BLLS & PARAFAC 2 & MCR-ALS \\
\hline Direct fluorescence & & & & \\
Estimated concentration (ppm) & $4.35(0.41)$ & $4.94(0.16)$ & $4.51(0.44)$ & $4.76(0.18)$ \\
Recovery (\%) & 87.4 & 99.2 & 90.6 & 95.6 \\
Components number & 4 & $3(1+2$ interferents) & 4 & 5 \\
Derivatization fluorescence & & & $0.28(0.10)$ & $0.29(0.11)$ \\
Estimated concentration $(\mathrm{ppm})$ & $0.29(0.11)$ & $0.31(0.0012)$ & 93.3 & 96.7 \\
Recovery (\%) & 96.7 & 103.3 & 4 & 4 \\
Components number & 5 & $3(1+2$ interferents $)$ & 4 & \\
Standard USP HPLC-UV $(n=3)$ & & &
\end{tabular}

A three component model was used to model the Verapamil drug EEM obtained by derivatization fluorescence with PARAFAC and PARAFAC2. With these models, the predicted concentration and described recoveries of the expected Verapamil concentration $(0.30 \mathrm{ppm})$ were $0.28 \mathrm{ppm}$ and $93.3 \%$ with PARAFAC; $0.284 \mathrm{ppm}$ and 94.7\% with PARAFAC2. With MCR-ALS four component model a predicted concentration of $0.30 \mathrm{ppm}$ and a recovery of $100.0 \%$. When BLLS was applied, taken into account one component and two interferents in the system, an estimation of $0.30 \mathrm{ppm}$ and a recovery of $100.0 \%$ were found. With the derivatization procedure, similarly to that observed in the direct analysis, the best estimation of the four methods was also found with MCR-ALS and BLLS methods.

The Fig. 4 presents the excitation and emission spectra estimated with the models that allows the best Verapamil concentration estimation: (a) with a three components non negativity constraint PARAFAC model; (b) with a one component and two interferents system BLLS model; (c) with a three components non negativity constraint in the first and third dimensions PARAFAC2 model; (d) with a four components non negativity constraint MCR-ALS model. For all the models three of the components correspond to the fluorescence of the derivatization products, and the four component corresponds to a background signal.

The figures of merit calculated are shown in Table 5. By the analysis of this table it is possible to see that similar results were found for the figures of merit by the two models. Even so a greater sensitivity was found by the BLLS model and greater selectivity by the PARAFAC model. Similar LODs were found with the two models.

\section{Pharmaceutical formulation quantification}

Table 6 shows the results of the analysis of Verapamil in a pharmaceutical formulation using the four decomposition methods. A greater number of components it is generally needed in order to obtain good concentrations estimations which is due to the existence of a more complex background. In the direct fluorescence approach, four components have been used for PARAFAC and PARAFAC2, three components for BLLS (one component and two interferents), and five components for MCR-ALS. In the derivatized procedure, five components have been used for PARAFAC, three components for BLLS (one component and two interferents) and four components for PARAFAC2 and MCR-ALS. A global comparison of the results shows that, taking as reference the recovery obtained by the USP HPLC-UV method (93.6\%), the best quantification result is found by direct fluorescence spectroscopy with the MCR-ALS model (95.6\%) and by derivatization fluorescence spectroscopy with the PARAFAC2 model $(93.3 \%)$.

\section{Conclusions}

The results obtained by the different three-dimensional decomposition methods allow to conclude that the EEM of Verapamil drug obtained by direct fluorescence has marked deviations from the trilinear structure, and the EEM obtained by derivatization fluorescence spectroscopy has an approximately trilinear structure. The deviations of the EEM to the trilinear model are essentially due to the presence of scatter bands.

For the two EEM structures under investigation the highest model fit is observed with MCR-ALS. In the analysis of the EEM obtained by direct fluorescence spectroscopy at least a two components PARAFAC, BLLS and MCR-ALS models and a three components PARAFAC2 model are needed. In the analysis of the EEM structures obtained by derivatization fluorescence spectroscopy at least a three components PARAFAC, BLLS and PARAFAC2 and a four components MCR-ALS models are needed. The limit of detection found in the derivatized procedure is lower than in the direct procedure. The best model estimations in the Verapamil drug are found by the MCR-ALS and BLLS models. Worse model estimations are 
obtained for the analysis of the EEM obtained by direct fluorescence spectroscopy when compared with the derivatization procedure.

The best estimations for the Verapamil concentration in the pharmaceutical formulation were obtained by direct fluorescence spectroscopy with the MCR-ALS model and by derivatization fluorescence spectroscopy with the PARAFAC2 model. Also, these concentration estimations agree with those obtained with the USP HPLC-UV reference method.

\section{References}

1. Lakowicz JR (2006) Principles of fluorescence spectroscopy, 3rd edn. Springer, New York

2. Sharma A, Schulman SG (1999) Introduction to fluorescence spectroscopy, 1st edn. Wiley, New York

3. Smilde AK, Bro R, Geladi P (2004) Multi-way analysis: applications in the chemical sciences, 1st edn. Wiley, New York

4. Bro R (2006) Review on multiway analysis in chemistry-2000 2005. Crit Rev Anal Chem 36(3-4):279-293

5. Escandar GM, Faber NKM, Goicoechea HC, Muñoz de la Peña A, Olivieri AC, Poppi RJ (2007) Second- and third-order multivariate calibration: data, algorithms and applications. Trends Anal Chem 26(7):752-765

6. Rinnan $\AA$, Andersen CM (2005) Handling of first order Rayleigh scatter in PARAFAC modelling of fluorescence excitationemission data. Chemometr Intell Lab Syst 76(1):91-99

7. Leurgans S, Ross R (1992) Multilinear models: applications in spectroscopy. Stat Sci 7(3):289-310

8. Bro R, Kiers HAL (2003) A new efficient method to determining the number of components in PARAFAC models. J Chemometr 17(5):274-286

9. Booksh KS, Kowalski BR (1994) Theory of analytical chemistry. Anal Chem 66(15):782A-791A

10. The United States Pharmacopeia (2007) USP 30. NF 25, United States Pharmacopeial Convention Ed. - The National Formulary

11. Esteves da Silva JCG, Leitão JMM, Costa FS, Ribeiro JLA (2002) Detection of verapamil drug by fluorescence and trilinear decomposition techniques. Anal Chim Acta 453(1):105-115

12. Harshman RA (1970) Foundations of the PARAFAC procedure: models and conditions for an "explanatory" multi-mode factor analysis. UCLA Work Pap Phon 16:1-84

13. Bro R (1997) PARAFAC. Tutorial and applications. Chemometr Intell Lab Syst 38(2):149-171

14. Linder M, Sundberg R (1998) Second-order calibration: bilinear least squares regression and a simple alternative. Chemom Intell Lab Syst 42(1-2):159-178

15. Linder M, Sundberg R (2002) Precision of prediction in secondorder calibration, with focus on bilinear regression methods. J Chemometr 16(1):12-27

16. Harshman RA (1972) PARAFAC2: mathematical and technical notes. UCLA Work Pap Phon 22:30-44

17. Kiers HAL, Berge JMFT, Bro R (1999) PARAFAC2-Part I. A direct fitting algotithm for the PARAFAC2 model. J Chemometr 13(3-4):275-294

18. Tauler R (1995) Multivariate curve resolution applied to second order data. Chemometr Intell Lab Syst 30(1):133-146

19. Saurina J, Tauler R (2000) Strategies for solving matrix effects in the analysis of triphenyltin in sea-water samples by three-way multivariate curve resolution. Analyst 125(11):2038-2043
20. Frenich AG, Zamora DP, Vidal JLM, Galera MM (2001) Resolution (and quantitation) of mixtures with overlapped spectra by orthogonal projection approach and alternating least squares. Anal Chim Acta 449(1-2):143-155

21. Stedmon CA, Markager S, Bro R (2003) Tracing dissolved organic matter in aquatic environments using a new approach to fluorescence spectroscopy. Mar Chem 82(3-4):239-254

22. Cory RM, McKnight DM (2005) Fluorescence spectroscopy reveals ubiquitous presence of oxidized and reduced quinones in dissolved organic matter. Environ Sci Technol 39(21):81428149

23. Holbrook RD, Yen JH, Grizzard TJ (2006) Characterizing natural organic material from the Occoquan Watershed (Northern Virginia, US) using fluorescence spectroscopy and PARAFAC. Sci Total Environ 361(1-3):249-266

24. Kim Y, Jordan JA, Nahorniak ML, Booksh KS (2005) Photocatalytic degradation-excitation-emission matrix fluorescence for increasing the selectivity of polycyclic aromatic hydrocarbon analyses. Anal Chem 77(23):7679-7686

25. Goicoechea HC, Yu S, Olivieri AC, Campiglia AD (2005) Fourway data coupled to parallel factor model applied to environmental analysis: determination of 2,3,7,8-tetrachloro-dibenzo-para-dioxin in highly contaminated waters by solid-liquid extraction laserexcited time-resolved Shpol'skii spectroscopy. Anal Chem 77 (8):2608-2616

26. Nahorniak ML, Cooper GA, Kim Y, Booksh KS (2005) Three- and four-way parallel factor (PARAFAC) analysis of photochemically induced excitation-emission kinetic fluorescence spectra. Analyst 130(1):85-93

27. Antunes MCG, Esteves da Silva JCG (2005) Multivariate curve resolution analysis excitation-emission matrices of fluorescence of humic substances. Anal Chim Acta 546(1):5259

28. Esteves da Silva JCG, Tavares MJCG, Tauler R (2006) Multivariate curve resolution of multidimensional excitation-emission quenching matrices of a Laurentian soil fulvic acid. Chemosphere 64(11):19391948

29. Nikolajsen RPH, Booksh KS, Hansen ÅM, Bro R (2003) Quantifying catecholamines using multi-way kinetic modelling. Anal Chim Acta 475(1-2):137-150

30. Olivieri AC, Arancibia JA, Muñoz de la Peña A, Durán-Merás I, Mansilla AE (2004) Second-Order advantage achieved with fourway fluorescence excitation-emission-kinetic data processed by parallel factor analysis and trilinear least-squares. determination of methotrexate and leucovorin in human urine. Anal Chem 76 (19):5657-5666

31. Arancibia JA, Escandar GM (2003) Two different strategies for the fluorimetric determination of piroxicam in serum. Talanta 60 (6): 1113-1121

32. Rodríguez-Cuesta MJ, Boqué R, Rius FX, Zamora DP, Galera MM, Frenich AG (2003) Determination of carbendazim, fuberidazole and thiabendazole by three-dimensional excitation-emission matrix fluorescence and parallel factor analysis. Anal Chim Acta 491 (1):47-56

33. Christensen J, Becker EM, Frederiksen CS (2005) Fluorescence spectroscopy and PARAFAC in the analysis of yogurt. Chemometr Intell Lab Syst 75(2):201-208

34. Guimet F, Ferré J, Boqué R, Rius FX (2004) Application of unfold principal component analysis and parallel factor analysis to the exploratory analysis of olive oils by means of excitationemission matrix fluorescence spectroscopy. Anal Chim Acta 515 (1):75-85

35. MATLAB 6.0, The Math Works Inc., Natick, MA, USA

36. Olivieri AC, Goicoechea HC, Iñón FA (2004) MVC1: an integrated MatLab toolbox for first-order multivariate calibration. Chemom Intell Lab Syst 73(2):189-197 
37. Lorber A (1986) Error propagation and figures of merit for quantification by solving matrix equations. Anal Chem 58(6):1167-1172

38. Messick NJ, Kalivas JH, Lang PM (1996) Selectivity and related measures for nth-order data. Anal Chem 68(9):1572-1579

39. Faber NM, Ferre J, Boqué R, Kalivas JH (2002) Second-order bilinear calibration: the effects of vectorising the data matrices of the calibration set. Chemom Intell Lab Syst 63(2):107-116
40. Olivieri AC, Faber NM (2004) Standard error of prediction in parallel factor analysis of three-way data. Chemom Intell Lab Syst 70(1):75-82

41. Rodríguez LC, Campaña AG, Linares CJ, Ceba MR (1993) Estimation of performance characteristics of an analytical method using the data set of the calibration experiment. Anal Lett 26 (6): $1243-1258$ 